\title{
Prevalence of Intimate Partner Violence and Beliefs About Partner Violence Screening Among Young Men
}

\author{
Tova B. Walsh, $P b D^{1}$ \\ Rita C. Seabrook, $P b D^{2}$ \\ Richard M. Tolman, $P h D^{3}$ \\ Shawna J. Lee, $P b D^{3}$ \\ Vijay Singh, $M D^{4}$ \\ 'School of Social Work, University \\ of Wisconsin, Madison, Wisconsin \\ ${ }^{2}$ Institutional Analysis, University \\ of Chicago, Chicago, Illinois \\ ${ }^{3}$ School of Social Work, University \\ of Michigan, Ann Arbor, Michigan \\ ${ }^{4}$ Departments of Emergency, Family, \\ and Internal Medicine, Medical School, \\ University of Michigan, Ann Arbor, \\ Michigan
}

\begin{abstract}
PURPOSE Few clinical guidelines focus on how physicians can identify intimate partner violence (IPV) perpetration or victimization among male patients, and litthe is known of men's experiences and beliefs regarding screening in health care settings. Our objective was to determine prevalence of men's experiences with IPV screening in health care settings and associations with men's beliefs regarding health care clinician identification of IPV.
\end{abstract}

METHODS Using a cross-sectional online survey of a nationally representative sample of 916 men aged 18-35 years, we conducted survey-weighted descriptive analyses to determine IPV prevalence, screening experiences and beliefs, and multivariate logistic regression to examine associations of demographics, IPV perpetration, and IPV victimization with men's screening experiences and beliefs.

RESULTS Of 916 men surveyed, 19\% reported perpetration and 27\% reported victimization in relationship with current or previous spouse/partner, $90 \%$ believed health care clinicians should ask about perpetration, $92 \%$ believed health care clinicians should ask about victimization, but only $11 \%$ had been asked about perpetration and $13 \%$ about victimization. Beliefs regarding IPV were associated with African American non-Hispanic race, IPV perpetration, and IPV victimization. Experiences being asked about IPV were associated with educational attainment and IPV perpetration.

CONCLUSIONS Among young US men, 9 in 10 support IPV identification by health care clinicians, nearly 1 in 5 report using IPV, but only about 1 in 10 report health care clinicians asking about IPV. These represent missed opportunities for health care IPV identification. Beliefs and experiences regarding health care IPV identification vary by race, education, and men's IPV perpetration and victimization. These disparities can inform tailored health care identification approaches.

Ann Fam Med 2020;18:303-308. https://doi.org/10.1370/afm.2536.

\section{INTRODUCTION}

I ntimate partner violence (IPV) is a significant public health problem in the United States. Few clinical guidelines ${ }^{1-3}$ have focused on identifying male perpetration or victimization, yet 1 in 5 men report using physical force toward an intimate partner, ${ }_{1}^{4}$ and nearly 1 in 3 men report that an intimate partner has used physical force towards them. ${ }^{5}$ Health care settings hold potential for IPV identification ${ }^{6}$ and response among men. ${ }^{7}$ Given that IPV-involved men seek routine health care services ${ }_{1}^{4}$ primary care physicians have the opportunity to ask male patients about IPV perpetration ${ }^{3}$ or victimization. ${ }^{8}$ Increased identification, intervention, and referrals for male patients may help to reduce IPV and associated adverse health outcomes. ${ }^{9}$ Despite high male IPV prevalence and the existence of clinical recommendations to assist health care clinicians in asking men about IPV, it is unknown how frequently US men are asked by health care clinicians about IPV, and it is unclear if US men would even support being asked about IPV by health care clinicians.

\section{CORRESPONDING AUTHOR}

Tova B. Walsh, PhD

School of Social Work

University of Wisconsin

1350 University Avenue

Madison, WI 53706

tbwalsh@wisc.edu 
Our objective was to use a nationally representative sample of young US men to determine (1) prevalence of young men's IPV involvement, and beliefs and experiences with health care IPV identification, and (2) demographic and IPV history associations of young men's beliefs about whether health care clinicians should ask male patients about IPV perpetration or victimization, and men's experiences being asked by a health care clinician about IPV perpetration or victimization. This knowledge can help guide development of tailored identification approaches and responses for male patients who have experienced IPV and potentially prevent violence by men toward their partners.

\section{METHODS}

\section{Participants}

The Men's Health, Fatherhood, and Relationships Study is a cross-sectional survey of men aged 18-35 years administered online from August through September 2014 using the KnowledgePanel (Ipsos), a probability-based web panel designed to be representative of the civilian, non-institutionalized US population. ${ }^{10}$ Panel members are chosen though random-digit dialing and address-based sampling, and households without Internet access are provided with a web-enabled device and free Internet service. KnowledgePanel sent 2 e-mail reminders and provided 1 telephone reminder during the survey administration. KnowledgePanel uses an incentive structure with panel participants that includes raffles with cash and other prizes. The first screen of the online survey provided participants with self-read informed consent. The University of Michigan Health Sciences and Behavioral Sciences Institutional Review Board approved the study.

\section{Measures}

IPV perpetration and victimization were measured using physical violence items from the Conflict Tactics Scale (CTS) for Partner and Spouse ${ }^{11}$ as adapted for use by the National Comorbidity Survey. ${ }^{12-13}$ Participants were presented with a list of behaviors from the CTS minor and severe physical violence subscales, and they were asked how frequently they had done each of the things on the list to their current or previous spouse/partner and how frequently their current or previous spouse/partner had done each thing to them. The minor physical violence subscale included items such as "pushed, grabbed, or shoved, threw something; slapped or hit," and the severe physical violence subscale included items such as "kicked, bit,

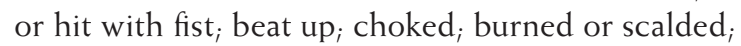
threatened with knife or gun." Participants reported frequency using a 4-point Likert-type scale from
1 (never) to 4 (often). IPV perpetration and victimization were dichotomized into no IPV perpetration or victimization (responded never to all items) vs any IPV perpetration or victimization (responded with a value greater than 1 to any number of items) for the purpose of analysis. IPV was further defined as perpetration only, victimization only, and both perpetration and victimization.

Beliefs about health care clinician IPV identification were assessed by asking if health professionals should ask their patients about whether they had hurt or frightened a partner (perpetration), or had been hurt or frightened by a partner (victimization). ${ }^{14}$ Experiences with health care clinician IPV identification were assessed with questions addressing whether they had ever been asked by a health professional if they had hurt or frightened a partner (perpetration), or been hurt or frightened by a partner (victimization). ${ }^{14}$ Items assessing beliefs and experiences were previously used in a UK study on asking men about IPV in a family medicine context. ${ }^{14}$

Sociodemographic questions included respondent's age, household income, education level, and race/ ethnicity.

\section{Analysis Plan}

All analyses were conducted using Stata version 15.1 (StataCorp LLC). When properly weighted, the data collected via the KnowledgePanel yields nationally representative estimates for non-incarcerated adult men aged 18 to 35 years. For each measure, we created nationally representative estimates by applying survey weights that accounted for the sampling design and missing data (no response to question). Measures were weighted based on sex, age, race, education, census region, annual household income, home ownership status, metropolitan area (yes/no), and Internet access (yes/no) from the most recent (relative to time of data collection) March 2013 Annual Social and Economic Supplement of the Current Population Survey.

The response rate was $47 \%(1,346 / 2,889)$, and differences in responders and nonresponders were addressed by survey weighting. Of 1,346 men who completed the survey, only those who reported ever having been in a romantic relationship $(79.6 \%$, $\mathrm{n}=1,072$ ) were asked questions about IPV perpetration. Most of these men indicated they had been in romantic relationships with women only $(93.6 \%$, $\mathrm{n}=985$ ). The analysis sample includes 916 respondents who reported ever having been in a romantic relationship and had complete data on all variables. Because we analyzed a subpopulation of the full sample, we used Stata's subpop command, which correctly calculates standard errors. 
We measured descriptive statistics for each variable. Victims only, perpetrators only, and victim-perpetrators (men who reported both victimization and perpetration) were included as separate groups in descriptive analyses. The perpetrator-only group was too small for further analysis, so 2 groups were included in regression models: men who reported any perpetration and men who reported any victimization.

We next conducted 4 separate multivariate logistic regressions: beliefs regarding health care identification of IPV perpetration, beliefs regarding health care identification of IPV victimization, experiences with health care identification of IPV perpetration, and experiences with health care identification of IPV victimization. Age, income, education, race/ethnicity, IPV perpetration, and IPV victimization served as independent variables that were entered into each model in 1 step. We calculated odds ratios and $95 \%$ CIs of the odds ratios in order to determine statistical significance at the $\alpha=.05$ level.

\section{RESULTS}

Sample characteristics are presented in Table 1. Of note, though the vast majority of young men believed health care clinicians should ask male patients about IPV perpetration (89.5\%) and victimization (92.1\%), only a minority of young men had ever been asked about IPV perpetration (11\%) or victimization (13\%). Also of note, of those men with any form of IPV involvement (perpetration only, victimization only, or both perpetration and victimization $\mathrm{n}^{\mathrm{n}}=271$ ), the majority $(56 \%, 152 / 271)$ reported both perpetration and victimization.

Predictors of beliefs and experiences regarding being asked by a health care clinician about IPV are reported in Table 2. Men with lower education were more likely to report being asked about IPV victimization, and African American non-Hispanic men were less likely to believe that health care clinicians should ask male patients about IPV victimization. Men who had perpetrated IPV were less likely to believe that health care clinicians should ask male patients about IPV perpetration or victimization. Men who had been victims of IPV were more likely to believe that clinicians should ask male patients about victimization. Men who had perpetrated IPV were more likely to report being asked about perpetration.

\section{DISCUSSION}

Though primary care physicians may face barriers to identifying or responding to IPV among men, ${ }^{15}$ approximately 9 in 10 young men in the United States

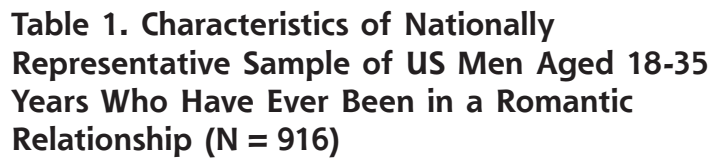

Table 1. Characteristics of Nationally Representative Sample of US Men Aged 18-35 Years Who Have Ever Been in a Romantic Relationship ( $\mathrm{N}=916)$

\begin{tabular}{|c|c|}
\hline Characteristic & Value $^{a}$ \\
\hline Age, mean (SD), y & $27(5.3)$ \\
\hline \multicolumn{2}{|l|}{ Annual income, (No. \%), \$ } \\
\hline $0-24,999$ & $108(11.8)$ \\
\hline $25,000-49,999$ & $212(23.2)$ \\
\hline $50,000-74,999$ & $203(22.2)$ \\
\hline $75,000-99,999$ & $182(19.9)$ \\
\hline $100,000+$ & $210(23.0)$ \\
\hline \multicolumn{2}{|l|}{ Education, (No. \%) } \\
\hline Less than high school & $90(9.9)$ \\
\hline High school & $266(29.0)$ \\
\hline Some college & $318(34.8)$ \\
\hline Bachelor's degree or higher & $241(26.3)$ \\
\hline \multicolumn{2}{|l|}{ Race, (No. \%) } \\
\hline White, non-Hispanic & $564(61.6)$ \\
\hline African American, non-Hispanic & $92(10.1)$ \\
\hline Hispanic & $201(22.0)$ \\
\hline Other, non-Hispanic & $59(6.4)$ \\
\hline \multicolumn{2}{|l|}{ IPV perpetration or victimization, (No. \%) } \\
\hline Any physical perpetration & $176(19.2)$ \\
\hline Any physical victimization & $247(27.0)$ \\
\hline Perpetration only & $24(2.6)$ \\
\hline Victimization only & $95(10.4)$ \\
\hline Both perpetration and victimization & $152(16.6)$ \\
\hline Neither perpetration nor victimization & $645(70.4)$ \\
\hline \multicolumn{2}{|c|}{$\begin{array}{l}\text { Belief that health care clinicians should ask male } \\
\text { patients about IPV, (No. \%) }\end{array}$} \\
\hline Perpetration & $820(89.5)$ \\
\hline Victimization & $844(92.1)$ \\
\hline \multicolumn{2}{|c|}{$\begin{array}{l}\text { Experiences being asked by a health care clinician } \\
\text { about IPV, (No. \%) }\end{array}$} \\
\hline Perpetration & $101(11.0)$ \\
\hline Victimization & $119(13.0)$ \\
\hline
\end{tabular}

supported being asked by a health care clinician about IPV perpetration or victimization. Although nearly 1 in 5 young men reported any use of IPV towards their partners and over 1 in 4 young men reported use of IPV by their partners towards them, only about 1 in 10 had been asked by a health care clinician about perpetration or victimization. These gaps demonstrate missed opportunities for IPV identification, as well as needed interventions and referrals. Education, race/ ethnicity, and history of involvement in physical IPV as perpetrator or victim were linked to beliefs about or experiences with being asked by a health care clinician about IPV among this nationally representative sample of young men. Understanding these associations may 
aid primary care physicians in identifying male patients who have been perpetrators or victims of IPV and addressing their needs for mental and behavioral health services or referral to IPV advocacy organizations for intervention.

Young men aged 18-35 years tend to be healthy, raising questions about how often they would be seen in a primary care practice and how much opportunity a physician would have to screen them for IPV. In the current sample, $60 \%$ of men reported having at least 1 regular doctor. While men are more likely than women, and younger adults are more likely than older adults, to seek health care on an as needed rather than preventative basis, ${ }^{16-18}$ our data show that $60 \%$ of young men have a regular doctor. When young men seek health care with that primary care physician, those health care encounters offer opportunities to identify IPV.

Primary care physicians can be mindful of distinct patient needs and resultant opportunities to address IPV among a broad range of male patients. A larger age distribution might reveal cohort effects and reflect shifting social norms about gender roles and relationships.

This study focused on young men, known to have higher rates of past year violence than older men, ${ }^{19,20}$ and examined the opportunity for primary care physicians to intervene early with men who experience and/or perpetrate IPV to prevent continuing or subsequent violence.

Further research with sufficiently large sample sizes in each group is needed to examine potential variation in characteristics associated with experiences and beliefs about health care clinician identification of IPV among men who are victims only, perpetrators only, or both victims and perpetrators of IPV. It is particularly important that future research examine group differences given that extant research has shown that victim-perpetrators are at significantly higher risk for negative health outcomes compared to victims only or perpetrators only. ${ }^{21}$

Given that $16.6 \%$ of the young men in our sample reported experiencing both perpetration and victimization, and given that the majority of young men who disclosed IPV involvement reported both perpetration and victimization, assessment for both perpetration and victimization is needed for primary care physicians to determine the different service needs of male patients with IPV. The current study demonstrates that asking men about IPV perpetration and victimization is feasible and acceptable, and this finding raises the question of effective responses. Responses may include counseling, brief interventions, and referrals, though these interventions have not been tested in a primary care setting. ${ }^{15}$ Brief motivational interviewing interventions focused on IPV perpetration have been shown to be effective in hospital and emergency department settings, ${ }_{1}^{22,23}$ but these interventions have not been examined in primary care settings. Further exploration is needed to identify effective treatments in primary care settings.

Routine identification may improve management of IPV, ${ }^{24}$ but tailored identification and intervention 
Experiences Being Asked by a Health Care Clinician About IPV

\begin{tabular}{|c|c|c|c|}
\hline \multicolumn{2}{|c|}{ Perpetration } & \multicolumn{2}{|c|}{ Victimization } \\
\hline$\%$ (mean) & AOR $(95 \% \mathrm{Cl})$ & $\%$ (mean) & AOR $(95 \% \mathrm{Cl})$ \\
\hline $27(5.1)$ & $1.01(0.96,1.06)$ & $27(5.2)$ & $1.01(0.96,1.06)$ \\
\hline $12(11.2)$ & $1.27(0.51,3.20)$ & $16(14.5)$ & $.86(0.38,1.94)$ \\
\hline $24(11.2)$ & $1.25(0.58,2.69)$ & $36(16.7)$ & $1.07(0.57,2.02)$ \\
\hline $16(7.8)$ & Reference & $26(12.6)$ & Reference \\
\hline $23(12.8)$ & $1.77(0.82,3.82)$ & 19 (10.5) & $0.83(0.39,1.75)$ \\
\hline $25(12.1)$ & $1.73(0.79,3.78)$ & $23(10.8)$ & $0.890 .43,1.81)$ \\
\hline $15(16.5)$ & $2.22(0.82,6.05)$ & $22(24.1)$ & $2.63(1.07,6.47)^{a}$ \\
\hline $28(10.5)$ & $1.55(0.76,3.17)$ & $36(13.7)$ & $1.53(0.81,2.90)$ \\
\hline $39(12.1)$ & $1.59(0.89,2.84)$ & $39(12.3)$ & $1.36(0.78,2.37)$ \\
\hline $19(7.9)$ & Reference & $21(8.8)$ & Reference \\
\hline $61(10.7)$ & Reference & 67 (11.9) & Reference \\
\hline $13(14.5)$ & $1.17(0.48,2.85)$ & $20(21.4)$ & $1.70(0.75,3.83)$ \\
\hline $22(11.2)$ & $1.01(0.56,1.79)$ & $27(13.3)$ & $1.03(0.59,1.79)$ \\
\hline $4(7.2)$ & $.64(0.17,2.45)$ & $5(9.1)$ & $.870(.27,2.76)$ \\
\hline $33(18.5)$ & $2.18(1.04,4.57)^{\mathrm{a}}$ & $31(17.9)$ & $1.49(0.71,3.11)$ \\
\hline $68(9.2)$ & Reference & $87(11.8)$ & Reference \\
\hline $36(14.4)$ & $0.90(0.44,1.82)$ & $37(15.0)$ & $0.95(0.49,1.84)$ \\
\hline $65(9.7)$ & Reference & $82(12.2)$ & Reference \\
\hline
\end{tabular}

methods may be needed to sensitively address beliefs among specific groups of men. Young men who disclosed IPV perpetration endorsed less support for male patients being asked about IPV, and this may reflect barriers to communication or lack of effective resources to address IPV perpetration. ${ }^{15}$ Further research is needed to assess best strategies for primary care physicians to use when identifying and responding to IPV among male patients. Primary care physicians have a unique position as community-based clinicians who can partner with local IPV services such as batterer intervention programs and advocacy organizations to respond to the needs of male patients identified with IPV perpetration or victimization.

Because our data are cross-sectional, only associations with, and not causation of, beliefs and experiences regarding IPV identification in health care settings can be determined. Data were collected in
2014, and additional research is needed to ascertain whether experiences and beliefs about partner violence screening among young men are changing over time. In this study we measured involvement in physical IPV only $y_{i}$ other forms of IPV including emotional abuse or sexual violence may be associated with other health care beliefs and experiences regarding IPV identification. IPV perpetration and victimization were measured by self-report of physical acts only and not about the context of these acts. The sample was overwhelmingly in heterosexual relationships. Additional research is needed to examine experiences and beliefs about partner violence screening among young men in nonheterosexual relationships.

Despite some limitations, our findings offer a first look at young men's beliefs regarding screening for IPV perpetration or victimization in health care settings and characteristics associated with beliefs and experiences being asked by a health care clinician about IPV. A substantial majority of this nationally representative sample of young men support health care clinician identification of both IPV perpetration and victimization, yet both are infrequently assessed in clinical practice. Given the potential to intervene in numerous negative health outcomes associated with IPV, primary care physicians can work to identify IPV perpetration and victimization among male patients and consider brief interventions or referrals for men identified with IPV.

To read or post commentaries in response to this article, see it online at https://www.AnnFamMed.org/content/18/4/303.

Submitted July 11, 2019; submitted, revised, December 21, 2019; accept4ed December 16, 2019.

Key words: intimate partner violence; men; primary health care

Funding support: This research was supported by the Robert Wood Johnson Foundation and Veterans Affairs Clinical Scholars Program at the University of Michigan.

Previous presentation: Presented at the Society for Social Work and Research Annual Conference; January 20, 2019; San Francisco, California.

\section{References}

1. Kimberg LS. Addressing intimate partner violence with male patients: a review and introduction of pilot guidelines. J Gen Intern Med. 2008;23(12):2071-2078.

2. Hegarty K, Forsdike-Young K, Tarzia L, Schweitzer R, Vlais R. Identifying and responding to men who use violence in their intimate relationships. Aust Fam Physician. 2016;45(4):176-181.

3. Penti B, Timmons J, Adams D. The role of the physician when a patient discloses intimate partner violence perpetration: a literature review. J Am Board Fam Med. 2018;31(4):635-644.

4. Singh V, Tolman R, Walton M, Chermack S, Cunningham R. Characteristics of men who perpetrate intimate partner violence. J Am Board Fam Med. 2014;27(5):661-668.

5. Smith SG, Zhang X, Basile KC, et al. The National Intimate Partner and Sexual Violence Survey: 2015 Data Brief - Updated Release. Atlanta, GA: National Center for Injury Prevention and Control, Centers for Disease Control and Prevention; 2018. 
6. Ernst AA, Weiss SJ, Morgan-Edwards S, et al. Derivation and validation of a short emergency department screening tool for perpetrators of intimate partner violence: the PErpetrator RaPid Scale (PERPS). J Emerg Med. 2012;42(2):206-217.

7. Tarzia L, Forsdike K, Feder G, Hegarty K. Interventions in health settings for male perpetrators or victims of intimate partner violence. Trauma Violence Abuse. 2020;21(1):123-137.

8. Curry SJ, Krist AH, Owens DK, et al; US Preventive Services Task Force. Screening for intimate partner violence, elder abuse, and abuse of vulnerable adults: US Preventive Services Task Force final recommendation statement. JAMA. 2018;320(16):1678-1687.

9. Rhodes KV, Houry D, Cerulli C, Straus H, Kaslow NJ, McNutt L-A. Intimate partner violence and comorbid mental health conditions among urban male patients. Ann Fam Med. 2009;7(1):47-55.

10. Gfk Infosheet. KnowledgePanel Methodology. https://osf.io/4shmq/ download. Accessed Jul 10, 2019

11. Straus MA. Measuring intrafamily conflict and violence: the conflict tactics scales. J Marriage Fam. 1979;41(1):75-88.

12. Kessler RC. National Comorbidity Survey: Baseline (NCS-1), 1990 1992. https://www.icpsr.umich.edu/icpsrweb/NAHDAP/studies/6693/ publications. Published 2008. https://doi.org/10.3886/ICPSR06693.v6.

13. Kessler RC. National Comorbidity Survey: Reinterview (NCS-2), 20012002. https://www.icpsr.umich.edu/icpsrweb/NAHDAP/studies/30921. Published 2016. https://doi.org/10.3886/ICPSR30921.v2.

14. Morgan K, Williamson E, Hester M, Jones S, Feder G. Asking men about domestic violence and abuse in a family medicine context: Help seeking and views on the general practitioner role. Aggress Violent Behav. 2014;19(6):637-642.

15. Penti B, Tran H, Timmons J, Rothman EF, Wilkinson J. Physicians' experiences with male patients who perpetrate intimate partner violence. J Am Board Fam Med. 2017;30(2):239-247.
16. Banks I, Baker P. Men and primary care: improving access and outcomes. Men's Health. 2013;4(5):39-41.

17. Schlichthorst M, Sanci LA, Pirkis J, Spittal MJ, Hocking JS. Why do men go to the doctor? Socio-demographic and lifestyle factors associated with healthcare utilisation among a cohort of Australian men. BMC Public Health. 2016;16(Suppl 3):1028.

18. Wang Y, Hunt K, Nazareth I, Freemantle N, Petersen I. Do men consult less than women? An analysis of routinely collected UK general practice data. BMJ Open. 2013;3(8):e003320.

19. Black MC, Basile KC, Breiding MJ, et al. The National Intimate Partner and Sexual Violence Survey (NISVS): 2010 Summary Report. Atlanta, GA: National Center for Injury Prevention and Control, Centers for Disease Control and Prevention; 2011.

20. Kann L, McManus T, Harris WA, et al. Youth Risk Behavior Surveillance - United States, 2017. MMWR Surveill Summ. 2018;67(8)(No. SS-8):1-114.

21. Ulloa EC, Hammett JF. The effect of gender and perpetrator-victim role on mental health outcomes and risk behaviors associated with intimate partner violence. J Interpers Violence. 2016;31(7):1184-1207.

22. Rothman EF, Wang N. A feasibility test of a brief motivational interview intervention to reduce dating abuse perpetration in a hospital setting. Psychol Violence. 2016;6(3):433-441.

23. Ngo QM, Eisman AB, Walton MA, et al. Emergency department alcohol intervention: effects on dating violence and depression. Pediatrics. 2018;142(1):e20173525.

24. Waalen J, Goodwin MM, Spitz AM, Petersen R, Saltzman LE. Screening for intimate partner violence by health care providers. Barriers and interventions. Am J Prev Med. 2000;19(4):230-237. 